\title{
Legislation in the genomic era: the Affordable Care Act and genetic testing for cancer risk assessment
}

\author{
Farzana L. Walcott, MD, $\mathrm{MPH}^{1,2}$ and Barbara K. Dunn, MD, PhD ${ }^{1}$
}

Originally intended to provide equitable access to basic healthcare services, the Affordable Care Act (ACA) has become a pioneer in the uncharted territory of genetic testing legislation as it applies to clinical practice. Heretofore, legislation pertaining to genetics has concerned genetic discrimination by employers and health insurance companies. ${ }^{1}$ The ACA, however, mandates private insurance coverage for specific preventive health services as outlined under grade A and B recommendations of the US Preventive Services Task Force (USPSTF) ${ }^{2}$ without cost sharing by the patient. In this manner, the law is defining what constitutes preventive health care and, as a natural consequence, subsequent clinical practice.

In this Commentary we address section 2713 of the ACA, "Coverage of Preventive Health Services," ${ }^{2}$ and the implications this section may have for genetic testing for cancer risk assessment. Here, the ACA refers to the definition of preventive health services as those services designated by the USPSTF with a grade $\mathrm{A}$ or $\mathrm{B}$ recommendation and those services that are supported by the Health Services and Resource Administration. Importantly, lack of a provision under the ACA does not equate to lack of coverage under insurance. It means only that private insurance is not required by law to provide a specific service to patients without cost sharing.

Based on a grade B designation by the USPSTF, ${ }^{3}$ genetic counseling and testing for hereditary breast and ovarian cancer (HBOC) syndrome for appropriate women is included under the ACA as a preventive service for women under essential health benefits. Consequently, private industry has begun to use the ACA in marketing campaigns, touting the "coverage" of $B R C A$ genetic testing for cancer risk assessment to healthcare providers. ${ }^{4}$ Under section 2713 of the ACA, however, this provision applies only to women who are unaffected by a personal history of BRCA-related cancers and who have a compelling family history of such cancers or an identified deleterious $B R C A$ mutation in family members. By default, then, this provision does not include (i) screening for other heritable cancer syndromes, (ii) women with a history of cancer, (iii) high-risk men, and (iv) risk-reducing interventions in the event a deleterious mutation is found on testing. Given that primary care providers will be guided by the USPSTF recommendations, especially those that are now highlighted under the ACA, it is critical to discuss the nuances of this legislation on genetic testing for cancer risk and potential sequelae of that testing.

Genetic counseling and testing for other, non-HBOC hereditary cancer syndromes is not a provision under ACA section 2713. Other autosomal dominant cancer syndromes affecting men and women, which may present with a spectrum of cancers similar to HBOC, are Lynch syndrome, Li-Fraumeni syndrome, and Cowden syndrome. Lynch syndrome is the most common hereditary colorectal cancer syndrome, accounting for $\sim 3-5 \%$ of all colorectal cancers diagnosed in the United States. ${ }^{5}$ Individuals with Lynch syndrome tend to develop colorectal cancer before age 50 , which is younger than the recommended age for initiating screening in standard populations. Lynch syndrome is also associated with high risks for endometrial and ovarian cancers. ${ }^{5}$ Similarly, Cowden syndrome is associated with increased risks of breast, thyroid, and endometrial cancers, and Li-Fraumeni syndrome with high risks of breast, brain, and soft-tissue cancers, among others. ${ }^{6}$ In fact, in early age-onset breast cancer (age $\leq 35$ years), the National Comprehensive Cancer Network guidelines support testing for $B R C A 1 / 2$ alongside TP53 testing for Li-Fraumeni syndrome either concurrently or after a negative $B R C A 1 / 2$ result. $^{5}$ This emphasizes the importance of having a focus in the ACA that expands beyond HBOC for genetic testing of hereditary cancer syndromes.

Women affected by breast and ovarian cancer are not guaranteed genetic counseling and testing without cost sharing, according to the ACA, section 2713. Current clinical practice according to the National Comprehensive Cancer Network guidelines ${ }^{5}$ prioritizes genetic testing for cancer-affected individuals before unaffected relatives. The rationale for this approach is that testing the cancer-affected individual first has a higher likelihood of obtaining an informative result for establishing the genetic basis for cancer in the family. In an individual without cancer but with a family history suggestive of an inherited cancer syndrome (and in whose family a definitive deleterious mutation has not been identified), a negative test result is less reassuring. It may mean that the individual has a mutation in another gene for which testing was not done or that there is a mutation in the family, but the individual tested did not inherit it.

${ }^{1}$ Chemoprevention Agents Research Development Group, Division of Cancer Prevention, National Cancer Institute, Rockville, Maryland, USA; ${ }^{2}$ Cancer Prevention Fellowship Program, Division of Cancer Prevention, National Cancer Institute, Rockville, Maryland, USA. Correspondence: Farzana L. Walcott (farzana.walcott@nih.gov) 
The USPSTF provides guidelines for primary care providers for the appropriate use of preventive interventions and screening in asymptomatic, average-risk populations. As such, the USPSTF states that the management of patients with cancer is beyond the scope of these recommendations. ${ }^{3}$ The identification of a germ-line mutation at the time of cancer diagnosis, however, may substantially influence surgical management. In patients with newly diagnosed breast cancer, multiple studies have shown that women who test positive for deleterious mutations in BRCA1/2 at the time of diagnosis are more likely to undergo simultaneous therapeutic surgery in the affected breast and risk-reducing surgery in the contralateral breast. This approach obviates the need for a second breast surgery for risk reduction and reduces the risk of contralateral breast cancer by $90 \%{ }^{7}$ Further, women, with or without a history of breast cancer, harboring a deleterious $B R C A 1 / 2$ mutation are at increased risk of developing ovarian cancer. This risk decreases considerably after prophylactic salpingo-oophorectomy and has substantial mortality benefits in BRCA1/2 mutation carriers. ${ }^{8}$ In light of these considerations the ACA section 2713 may benefit from expanded provisions for genetic testing as a preventive service for individuals with a previous diagnosis of cancer.

Men are not included in the USPSTF (and subsequently the ACA section 2713) recommendations for risk assessment for HBOC, though they may carry $B R C A 1 / 2$ or other germ-line mutations. HBOC is transmitted by autosomal dominant germline inheritance of a mutation that can be inherited via maternal or paternal lineage. Some studies indicate that prostate cancer in carriers of germ-line BRCA1/2 mutations may be more aggressive and present at a later stage than prostate cancers in noncarriers. ${ }^{5}$ Male breast cancer risk also increases significantly in men who have $B R C A 2$ mutations, and this risk increases with family history of breast and/or ovarian cancer or personal history of breast cancer. ${ }^{5}$ Documenting family history of all cancers, including gynecologic cancers and male breast cancer, with subsequent referral for appropriate genetic counseling and testing, is an important risk assessment intervention for men as well as women.

There are no provisions for risk-reducing interventions under ACA section 2713 in the event of a positive genetic test. Genetic testing for cancer risk assessment is becoming an integral component of preventive health practice. The ability to implement an intervention after a deleterious mutation is identified is critical to the ultimate value of the genetic test. For women with $B R C A 1 / 2$ mutations, prophylactic surgery and/ or yearly surveillance magnetic resonance imaging are costly interventions. ${ }^{5}$ None of these interventions is considered an essential health benefit for preventive care nor mandated by the ACA for coverage under private insurance-a gap in the ACA that has been noted by the Institute of Medicine. ${ }^{9}$ We recently discussed elsewhere ${ }^{10}$ how minorities and underinsured populations who underutilize genetic testing are particularly vulnerable to the aforementioned omissions of the ACA. This raises clinical management and ethical issues of whether, in accordance with the USPSTF and ACA, advising or offering genetic testing to individuals who may not be able to afford risk-reducing interventions in the event a deleterious mutation is identified is appropriate.

The ACA has prompted new dialogue regarding what is considered the scope of adequate preventive health care. In the past year publicity about the legal suit with Myriad Genetics over the patenting of $B R C A$ genes, together with Hollywood actress Angelina Jolie's announcement of her BRCA mutation-carrier status and subsequent bilateral mastectomies, have firmly fixed the public magnifying glass on women and genetic testing for HBOC. Meanwhile, other clinically important high-risk cancer syndromes continue to be overlooked. The keen public and clinical focus on HBOC may lead to genetic testing for BRCA1/2 mutations when testing for other syndromes with overlapping cancer presentations may be more appropriate, leading to a false sense of patient and provider reassurance in the event of a negative test result for BRCA1/2 mutations. Last, although genetic counseling is part of the USPSTF grade B recommendation for $B R C A$ risk assessment, women and men at risk for any type of hereditary cancer syndrome would benefit from this service, which provides information about potential outcomes of genetic testing, follow-up care, and even insurance coverage.

Cancer prevention, along with cancer genetics, is a nuanced practice based on multiple levels of risk assessment. Guidelines by professional organizations such as the National Comprehensive Cancer Network, American Society of Clinical Oncology, and Evaluation of Genomic Applications in Practice and Prevention, among others, might amplify our understanding of the recommended preventive practices surrounding genetic testing. Input from these additional specialty groups may stimulate better informed legislation and guidelines for genetic testing and insurance coverage to maximize potential benefits for this unique and evolving mode of preventive care.

\section{DISCLOSURE}

The authors declare no conflict of interest.

\section{REFERENCES}

1. Genetic Information Nondiscrimination Act (GINA), 2008. http://www genome.gov/24519851 and Executive Order 13145 Prohibiting the Use of Genetic Information in Hiring or Promoting, 2000. http://www.genome. gov/12513980 (websites accessed December 2014).

2. Patient Protection and Affordable Care Act. Public Law No. 111-148, Title I, Subtitle A, Section $2713(a)(1)$ and (a)(4-5), (2010). https://democrats. senate.gov/pdfs/reform/patient-protection-affordable-care-act-as-passed.pdf (accessed December 2014).

3. USPSTF A and B Recommendations, September 2014; Risk Assessment, Genetic Counseling, and Genetic Testing for BRCA-Related Cancer in Women, USPSTF, December 2013. http://www.uspreventiveservicestaskforce.org/ Page/Document/RecommendationStatementFinal/brca-related-cancer-riskassessment-genetic-counseling-and-genetic-testing (accessed December 2014).

4. Myriad Genetics says BRCA testing receives designation under Affordable Care Act. http://www.benzinga.com/news/13/03/3393791/myriad-geneticssays-brca-testing-receives-designation-under-affordable-care-act. 2013 (accessed December 2014).

5. National Comprehensive Cancer Network (NCCN) Clinical Practice Guidelines in Oncology: Genetic/Familial High-Risk Assessment: Breast and Ovarian. V I.2014. http://www.nccn.org/professionals/physician_gls/pdf/genetics_screening. pdf and NCCN Clinical Practice Guidelines in Oncology: Geneti/Familial HighRisk Assessment: Colorectal. V2.2014. http://www.nccn.org/professionals/ physician_gls/pdf/genetics_colon.pdf (accessed December 2014)

6. Lindor NM, McMaster ML, Lindor CJ, Greene MH; National Cancer Institute, Division of Cancer Prevention, Community Oncology and Prevention Trials 


\section{COMMENTARY}

Research Group. Concise handbook of familial cancer susceptibility syndromes second edition. J Natl Cancer Inst Monographs 2008;38:1-93.

7. Smith $\mathrm{KL}$, Isaacs $\mathrm{C}$. BRCA mutation testing in determining breast cancer therapy. Cancer J 2011;17:492-499.

8. Finch AP, Lubinski J, Møller P, et al. Impact of oophorectomy on cancer incidence and mortality in women with a BRCA1 or BRCA2 mutation. J Clin Oncol 2014;32:1547-1553.
WALCOTT and DUNN | The ACA and genetic testing for cancer risk assessment

9. Institute of Medicine (IOM). Appendix A: breast cancer. In: Clinical Preventive Services for Women: Closing the Gaps. National Academies Press: Washington, DC, 2011:188.

10. Walcott FL, Dunn BK, Deshields M, Baquet C. The Affordable Care Act and genetic testing for inheritable cancer syndromes: impact on high-risk underserved minorities. J Health Care Poor Underserved 2014;25(1 suppl): 46-62. 